\title{
Effects of sulfonamide antibiotics on digestion performance and microbial community during swine manure anaerobic digestion
}

\author{
Shaona Wang ${ }^{1}$, Kang $\mathrm{Du}^{2}$, Rongfang Yuan ${ }^{1^{\dagger}}$, Huilun Chen ${ }^{1}$, Fei Wang ${ }^{1}$, Beihai Zhou ${ }^{{ }^{\dagger}}$ \\ ${ }^{1}$ Beijing Key Laboratory of Resource-oriented Treatment of Industrial Pollutants, Department of Environmental Science and Engineering, School of \\ Energy and Environmental Engineering, University of Science and Technology Beijing, Beijing 100083, China \\ ${ }^{2}$ Beijing Municipal Research Institute of Environmental Protection, Beijing, 100037, China
}

\begin{abstract}
The effects of four types of sulfonamide antibiotics (SAs), including sulfaquinoxaline, sulfamethoxazole, sulfamethoxydiazine and sulfathiazole, on the digestion performance during anaerobic digestion process were studied using a lab-scale anaerobic sequencing batch reactor, and the changes of the community structure in the presence of SAs were investigated with the help of high throughput sequencing. The results indicated that when SAs were added, the hydrolytic acidification process was inhibited, and the accumulation of volatile fatty acids (VFAs) was induced, resulting in the suppression of methane production. However, the inhibition mechanism of different SAs was quite different. The inhibitory effect of high concentration of SAs on the hydrolysis of solid particulate matter into dissolved organic matter followed the order of sulfaquinoxaline $>$ sulfamethoxydiazine $>$ sulfathiazole $>$ sulfamethoxazole. SAs have obvious inhibitory effects on acidification and methanation of dissolved organic matter, especially sulfathiazole. The richness and the community composition of the microorganism including bacteria and archaea in the digestion system were affected by SAs. Under the effect of SAs, the relative abundance of many microorganisms is negatively correlated with methane production, among which Methanobrevibacter, a kind of Archaea, had the greatest influence on methane production.
\end{abstract}

Keywords: Anaerobic digestion, Sulfonamide antibiotics, Inhibition, Microorganism

\section{Introduction}

Anaerobic digestion is one of the main ways to treat pig manure [1], as it can not only reduce environmental pollution caused by manure, but also produce biomass energy $\left(\mathrm{CH}_{4}\right)[2,3]$. However, the addition of antibiotics in livestock and poultry farming can affect the anaerobic digestion process significantly [4]. As a broad-spectrum antibiotic, sulfonamide antibiotics (SAs) are often used in disease prevention and treatment in livestock and poultry industry [5]. With the increasing consumption of SAs, more and more attention has been paid to their effects on anaerobic digestion. According to the previous reports, the concentration of SAs such as sulfadimidine (SM2), sulfadiazine (SD), sulfamethoxazole (SMX) and sulfaquinoxaline (SQX) in livestock and poultry manure can range from $1.8 \mathrm{mg} / \mathrm{kg}$ to $91 \mathrm{mg} / \mathrm{kg}$ [6-8].

The anaerobic digestion process of feces is mainly based on

This is an Open Access article distributed under the term of the Creative Commons Attribution Non-Commercial License (http://creativecommons.org/licenses/by-nc/3.0/) which permits unrestricted non-commercial use, distribution, and reproduction in any medium, provided the original work is properly cited.

Copyright (C) 2021 Korean Society of Environmental Engineers a series of interlinked microbial processes including hydrolysis, digestion, acetogenesis and methanogenesis [9]. Antibiotics have negative influence on anaerobic digestion to produce biogas, and different kinds of antibiotics have different inhibitory effects on methane production process $[10,11]$. In terms of biogas production, the addition of sulfamethoxydiazine (SMD) in anaerobic digestion system largely determines the inhibition of methane production [12]. At the same time, the addition of SAs has different effects on different digestion processes. The inhibitory effect of three SAs, including sulfathiazole (STZ), Sulfamethazine (SMZ) and SMX on methane production ranged from 42 to $49 \%$ when the concentration of SAs was $25 \mathrm{mg} / \mathrm{L}$ [13]. Meanwhile, SAs can prevent the normal synthesis of dihydrofolic acid, thus inhibiting the growth and reproduction of bacteria. The accumulation of volatile fatty acids (VFAs) was not significantly affected when the concentration of SMX was below $40 \mathrm{mg} / \mathrm{L}$ [14]. SMZ is conducive to acidogenesis and acetogenesis

Received November 27, 2019 Accepted March 08, 2020

${ }^{\dagger}$ Corresponding author

Email: zhoubeihai@sina.com(Zhou), yuanrongfang@ustb.edu.cn(Yuan) Tel: +86-10-6233482(Zhou), +86-62334821(Yuan) Fax: 86-10-62334821 ORCID: 0000-0001-6137-395X 
processes, and it can destroy cell membranes to improve solubilization, and therefore improve VFAs production [15]. Because acetic acid is the main component of methane production, the content of acetic acid is the focus of attention in anaerobic process [16]. When SMZ was added into anaerobic system, methanogenesis was inhibited resulting in accumulation of acetic acid [15].

A variety of bacteria and archaea are involved in anaerobic digestion [17]. Microorganisms involved in this process have different metabolic needs, and there are many interrelationships among members of microbial communities [18]. Relative to archaea, hydrogenotrophic methanogens and acetoclastic methanogens were the main object of study. Compared with hydrogenotrophic methanogens, the concentration of antibiotics had greater impact on the species abundance and growth rate of acetoclastic methanogens [19]. Some SAs have little effect on archaea, but can inhibit the formation of ribosomal proteins and thus inhibit the proliferation of bacteria [20]. For bacteria, most hydrolysis and acidification processes depend on bacteria metabolism, which affects the stability of environmental factors in anaerobic digestion system [21]. Bauer's research showed that low concentration of antibiotics has little effect on the structure of microbial community, and increasing the concentration of antibiotics can reduce microbial diversity [22]. Although microbial communities exposed to antibiotics did not change significantly in species diversity indices, Syntrophobacter and Methanothrix associated with acetogenesis and acetoclastic methanogenesis, respectively [17]. In order to evaluate the results, Canonical Correspondence Analysis (CCA), as a statistical method, is also used to determine the relationship between biogas dynamics and microbial communities.

In this study, a laboratory-scale sequential batch fermenter was used to simulate biogas engineering conditions. The effects of sulfaquinoxaline (SQX), STZ, SMD, and SMZ on biogas production by anaerobic digestion were investigated. The inhibition mechanism of SAs was revealed by studying the changes of the environmental factors (VFAs, the total solid (TS) and the total organic carbon (TOC)) and the microbial community structure. In addition, CCA was used to find out the correlation among environmental factors and microbial community structure during anaerobic digestion.

\section{Material and Methods}

\subsection{Materials}

The swine manure used in this study was collected from a swine farm in Tianjin, China. The content of SQX, SMX, SMD and STZ in all samples was lower than the detection limit $(1 \mu \mathrm{g} / \mathrm{L})[23$, 24]. To improve the productivity of the biogas digester, fresh solid manure (collected with scrapers) from the pig barns with the total solid (TS) content of approximately $25 \%(w / w)$ was used as the influent of the biogas digester. In order to adjust the moisture content of the fermentation substrate, the TS was regulated to $10 \pm 0.5 \%$ with tap water. The inoculum (methanogens) was taken from digestion tank which had been running continuously in the laboratory for more than half a year at $37^{\circ} \mathrm{C}$ with a feedstock of swine manure (TS $=10 \%$ ). The basic characteristics of all initial substrates for anaerobic digestion are presented in Table S1 based on three tests in parallel.
SQX (purity $\geq 99 \%$ ), STZ (purity $\geq 99 \%$ ), SMX (purity $\geq 99 \%$ ) and SMD (purity $\geq 98 \%$ ) used in this study was purchased from Bailingwei Chemical Plant (Beijing, China). The standard stock solutions of SAs were prepared by dissolving each compound in methanol with a concentration of $10 \mathrm{~g} / \mathrm{L}$ and stored at $4^{\circ} \mathrm{C}$ in darkness. Before starting the experiment, the required stock solution was added to the digestion tank to dry methanol in the nitrogen stream, and then the corresponding substrates were added to restructure.

\subsection{Experimental Set-up}

A $1 \mathrm{~L}$ anaerobic reactor, with an inner diameter of $10 \mathrm{~cm}$ and a height of $15 \mathrm{~cm}$, was designed with a feeding inlet (internal diameter of $20 \mathrm{~mm}$ ) on the top and a digestate outlet (internal diameter of $20 \mathrm{~mm}$ ) at the side. The TS of swine manure and inoculum was adjusted to $10.0 \pm 0.3 \%$, and the SCOD of the fermentation substrate was determined to be $32.5 \pm 1.5 \%$. The initial $\mathrm{pH}$ was adjusted to $7.0 \pm 0.3$ with phosphate buffer solution. After sparged with nitrogen gas for $20 \mathrm{~min}$, all the one-time batch digester was capped with a rubber septum, and then inversely incubated in a shaking incubator with the speed of $60 \mathrm{rpm}$ at $37 \pm 0.2^{\circ} \mathrm{C}$. The effects of SAs (SQX, SMX, STZ and SMD) on anaerobic digestion at different concentrations $(0,5,10,50$ and $100 \mathrm{mg} / \mathrm{L})$ were studied. Three parallel experiments were set up in each group, and all the results were measured and analyzed with mean values. During the digestion, the biogas produced from each reactor was collected into gas bag for analysis. The volume was measured using a calibrated sampling syringe. Samples were taken every five days after the start of the experiment. During the sampling process, the anaerobic environment in the system was maintained using nitrogen flow. All samples are stored at $-20^{\circ} \mathrm{C}$ to keep them from being biological decomposition, and the frozen samples were thawed in the refrigerator at $4^{\circ} \mathrm{C}$ for $1 \mathrm{~d}$ before experimental analyses.

\subsection{Analytical Methods}

Volatile solid (VS) content was analyzed by drying the samples at $500^{\circ} \mathrm{C}$ for $4 \mathrm{~h}$, and TS content was obtained by drying the samples at $105^{\circ} \mathrm{C}$ for $12 \mathrm{~h}$ according to Gilcreas's research standard methods [25]. Solution chemical oxygen demand (SCOD) was analyzed according to the national standard code for Chinese characters (GB 11914-89) by using HACH digestion system and colorimetric spectrometer (DR6000, HACH, USA). The $\mathrm{pH}$ values of the samples were determined using a HQ30D $\mathrm{pH}$ meter (HACH, USA).

The concentrations of acetic acid (HAc), propionic acid (HPr), butyric acid (iso- $\mathrm{HBu}$ and $\mathrm{n}-\mathrm{HBu}$ ) and valeric acid (iso- $\mathrm{HVu}$ and $\mathrm{n}-\mathrm{HVu}$ ) were determined, and the total amount of VFAs was calculated. The VFAs concentration in samples was measured by a gas chromatograph (Shimadzu GC-2010) equipped with a flame-ionization detector and a $30 \mathrm{~m} \times 0.25 \mathrm{mmTRB}-\mathrm{FFAP}$ capillary column (film thickness $0.25 \mu \mathrm{m}$ ). Detailed methods refer to the methods of predecessors [4]. The elemental analyzer (Vario EL/micro cube, Germany) was used for determining the contents of $\mathrm{C}, \mathrm{H}$ and N. The values of TOC were analyzed by using a vario TOC meter (Elementar, Germany).

\subsection{Kinetic Fitting of Cumulative Methane Yield}

Linear, exponential and Gaussian fitting are usually used to evaluate 
the methane production performance of anaerobic digestion system. However, linear regression modules cannot accurately describe and predict cumulative methane production through the entire process, especially after the exponential phase [26]. To predict methane production potential during anaerobic digestion, the modified Gompertz equation was utilized to achieve representative simulations and predictions, as shown in Eq. (1).

$$
M_{\mathrm{t}}=M_{0} \exp \left(-\exp \left[\frac{R_{\mathrm{m}}}{M_{0}}\left(k^{\prime}-t\right)+1\right]\right)
$$

where $M_{\mathrm{t}}(\mathrm{mL} / \mathrm{g} \mathrm{VS})$ is the cumulative methane production at anaerobic digestion time $t(\mathrm{~d}), M_{0}(\mathrm{~mL} / \mathrm{g} \mathrm{VS})$ is the methane production potential, $R_{m}$ (mL/g VS)) is the maximum methane production rate, $k^{\prime}$ (d) is the lag phase time, $t$ is time (d), and $e$ is the Euler number which equals to 2.71828 .

\subsection{Microbial Community Analysis}

The communities of bacteria and archaea from fermented samples were analyzed using high-throughput sequencing performed on the MiSeq platform, in which the two terminal sequencing pair-end method was employed. Polymerase chain reactions of bacteria were performed with primers (341F-805R) [27] spanning the V3-V4 hypervariable region of the $16 \mathrm{~S}$ rRNA gene. Polymerase chain reactions of Archaea were performed with primers (349F- 806R) [28] spanning the V3-V4 hypervariable region of the 16S rRNA gene. Prior to sequencing on an Illumina MiSeq sequencing platform (Sangon Biotech Shanghai, China), the MOTHUR program was used to remove the chimeric sequences, which can pick operational taxonomic units (OTUs) with similarities greater than 97\%. The OTUs were classified using the alpha diversity metrics. The alpha diversity metrics were determined by comparing the smallest libraries for each sample. Alpha diversity analysis (Shannon and Simpson) was estimated to measure diversity. The abundance of unit sequence dataset of microorganism was expressed by its relative abundance, and the relative abundance among parallel groups was analyzed by average weight. The relationship between the environmental variables and the microbial community were investigated by Canonical Correspondence Analysis (CCA), which was calculated using CANOCO 4.5 software (Microcomputer Power, USA).

\section{Results and Discussion}

\subsection{Biogas Production Performance of Reactor}

\subsubsection{Biogas production rate and cumulative biogas yield}

Biogas production rate from anaerobic digestion under different concentrations of SAs is shown in Fig. S1. Biogas production rate of the anaerobic digestion process increased firstly and then decreased in all groups. It presents that the anaerobic digestion system could start up quickly and operated normally after inoculation. Among them, the group without SAs occurred the highest daily biogas production $(79 \mathrm{~mL} / \mathrm{gVS} \cdot \mathrm{d}$ ) on the second day after the start of the experiment. When SAs were added, the daily maximum gas production rate decreased and the occurrence time was delayed. When $5 \mathrm{mg} / \mathrm{L}$ and $100 \mathrm{mg} / \mathrm{L}$ SQX were added to anaerobic digestion system, the peak time of maximum gas production rate was delayed to the fourth and third days respectively, and the maximum gas production was also reduced to $69 \mathrm{~mL} / \mathrm{gVS} \cdot \mathrm{d}$ and $30 \mathrm{~mL} / \mathrm{gVS} \cdot \mathrm{d}$, respectively. The concentration of SQX had little effect on the time when the maximum biogas production rate appeared, but it caused a sharp decrease in the maximum daily gas production. This may be because the addition of SQX inhibited or killed some biogas-producing microorganisms. With the addition of SMX, different phenomena appeared in the production efficiency of biogas. When $5 \mathrm{mg} / \mathrm{L}, 10 \mathrm{mg} / \mathrm{L}, 50 \mathrm{mg} / \mathrm{L}$ and $100 \mathrm{mg} / \mathrm{L} \mathrm{SMX}$ were added to the system, the occurrence time of daily maximum gas production rate was delayed to the $4 \mathrm{~d}, 4 \mathrm{~d}, 6 \mathrm{~d}$ and $8 \mathrm{~d}$. However, the inhibition of daily maximum gas production rate was lower than that of SQX. In addition, adding SMX had a great influence on anaerobic digestion system at the initial stage, but with the prolongation of digestion time, the microorganisms in the system could adapt to SMX and reproduce normally [29]. It was also possible that SMX had a certain degradation effect in the anaerobic system [30], so that the content of SMX decreased and the system operated steadily.

From Fig. S1, when $5 \mathrm{mg} / \mathrm{L}$ SMD was added, no effect was found on daily gas production. However, when the concentration reached $10-50 \mathrm{mg} / \mathrm{L}$, the maximum daily gas production rate did not decrease significantly, but the occurrence time was delayed to the $6 \mathrm{~d}$ and $7 \mathrm{~d}$. Furthermore, when the concentration reached $100 \mathrm{mg} / \mathrm{L}$, the daily maximum gas production rate decreased rapidly, indicating that the high concentration of SMD had an irreversible effect on anaerobic digestion [31]. The effect of STZ on the anaerobic digestive system was similar to that of SQX, SMX and SMD. With the increase of SAs concentration, the daily maximum gas production rate decreased gradually. The occurrence time of daily maximum gas production rate was also postponed. When the concentration reached $100 \mathrm{mg} / \mathrm{L}$, the daily maximum gas production rate decreased by $70 \%$ compared with that without STZ. The STZ had a lag effect on the growth of microorganisms at low concentration, but it was reversible [32]. When the concentration exceeded a certain stage, it would have an irreversible effect on microorganisms.

In order to further understand the effect of SAs on anaerobic digestion total gas production, the gas production was accumulated (see Fig. 1). The biogas production process of the system mainly concentrates on the first $15 \mathrm{~d}$. The SAs had little effect on the cumulative biogas production at low concentration $(\leq 10 \mathrm{mg} / \mathrm{L})$. With the increase of SAs concentrations, the inhibition effect on biogas production of anaerobic digestion increased gradually, especially SQX and STZ. When the concentration of SQX in the digestion system was $50 \mathrm{mg} / \mathrm{L}$ and $100 \mathrm{mg} / \mathrm{L}$, the biogas yield was $43.1 \%$ and $48.6 \%$ decrease compared with the control group. When the concentration of STZ reached $100 \mathrm{mg} / \mathrm{L}$, the biogas yield decreased by 50.6\% compared with the matched group. Among all the experimental groups, STZ had the most obvious inhibition effect on biogas production process. The reason was that some microorganisms in anaerobic digestion system degraded STZ and produced derivatives, which were more toxic than STZ [33]. When the anaerobic digestion system contains high concentration of SMD and SMX, it can still maintain high gas production. Compared with the group without adding SAs, the cumulative biogas production was reduced by $30.4 \%$ and $20.9 \%$ respectively by adding $100 \mathrm{mg} / \mathrm{L} \mathrm{SMD}$ and SMX. On the one hand, SMD and SMX have strong degradation effect under anaerobic con- 

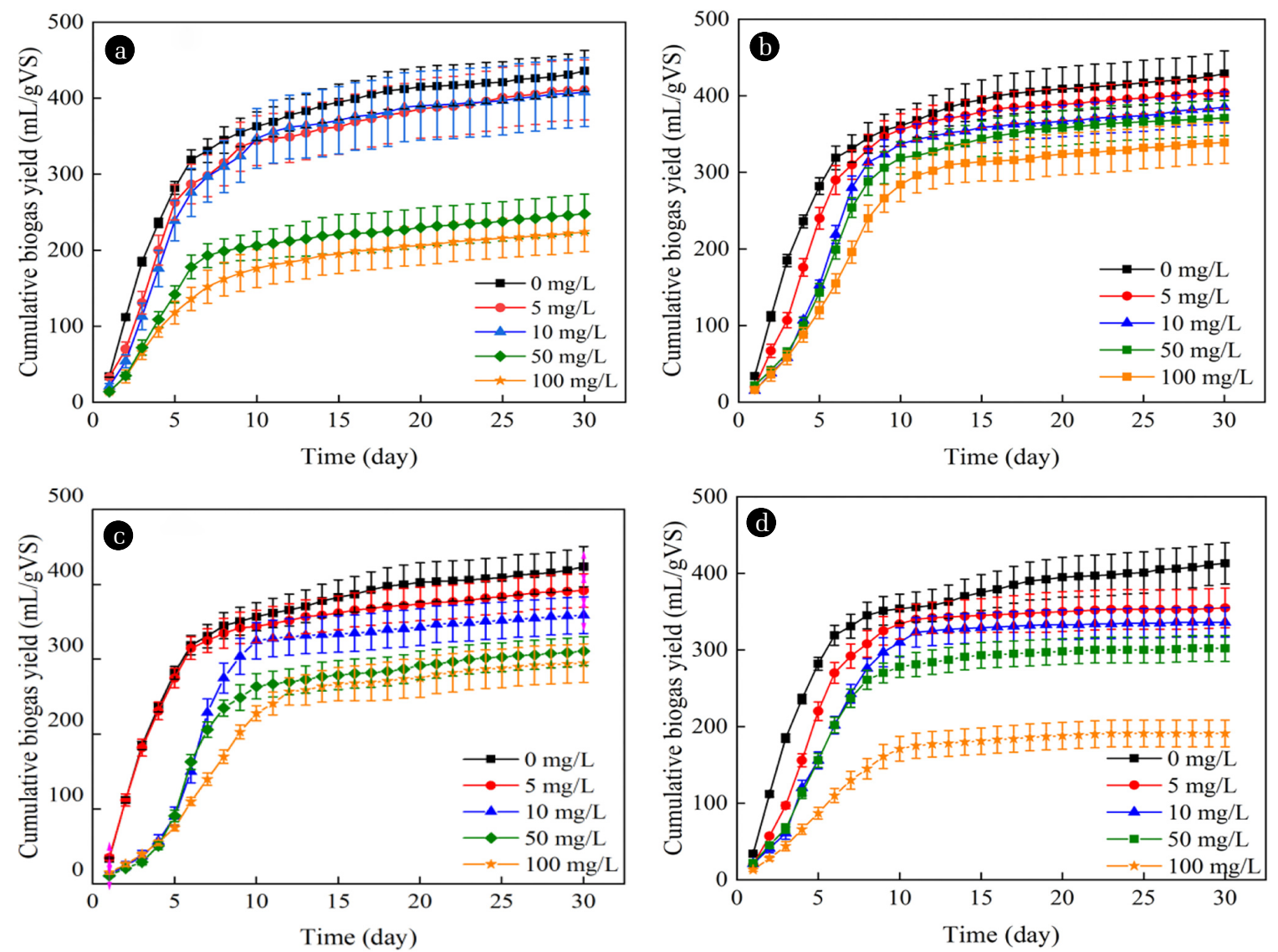

Fig. 1. Effect of SAs on cumulative biogas yield. (a)-(d) showed the effects of (a) SQX, (b) SMX, (c) SMD and (d) STZ on cumulative biogas yield in anaerobic digestion.

Table 1. Results of Kinetic Study Using Modified Gompertz Model.

\begin{tabular}{llccccc}
\hline & & $\mathbf{0 ~} \mathbf{~ m g} / \mathbf{L}$ & $\mathbf{5} \mathbf{~ m g} / \mathbf{L}$ & $\mathbf{1 0} \mathbf{~ m g} / \mathbf{L}$ & $\mathbf{5 0} \mathbf{~ m g} / \mathbf{L}$ & $\mathbf{1 0 0} \mathbf{~ m g} / \mathbf{L}$ \\
\hline \multirow{3}{*}{$\mathrm{SQX}$} & $M_{0}(\mathrm{~mL} / \mathrm{gVS})$ & $292.8 \pm 1.3$ & $276.9 \pm 1.2$ & $274.8 \pm 1.1$ & $163.6 \pm 1.2$ & $149.9 \pm 0.5$ \\
& $R_{\mathrm{m}}(\mathrm{mL} / \mathrm{gVS} \cdot \mathrm{d})$ & $37.2 \pm 1.8$ & $35.2 \pm 4.7$ & $36.3 \pm 3.8$ & $24.4 \pm 4.1$ & $15.6 \pm 0.7$ \\
& $k^{\prime}(\mathrm{d})$ & $0.45 \pm 0.09$ & $0.81 \pm 0.09$ & $1.07 \pm 0.08$ & $1.04 \pm 0.13$ & $1.87 \pm 0.01$ \\
\hline \multirow{3}{*}{$\mathrm{SMX}$} & $M_{0}(\mathrm{~mL} / \mathrm{gVS})$ & $291.8 \pm 1.3$ & $279.6 \pm 0.9$ & $265.8 \pm 1.8$ & $258.1 \pm 1.8$ & $236.9 \pm 4.2$ \\
& $R_{\mathrm{m}}(\mathrm{mL} / \mathrm{gVS} \cdot \mathrm{d})$ & $38.8 \pm 5.8$ & $41.8 \pm 3.5$ & $40.8 \pm 6.8$ & $33.8 \pm 5.4$ & $28.4 \pm 2.4$ \\
& $k^{\prime}(\mathrm{d})$ & $0.44 \pm 0.05$ & $1.18 \pm 0.06$ & $2.24 \pm 0.12$ & $1.93 \pm 0.13$ & $2.51 \pm 0.06$ \\
\hline \multirow{3}{*}{$\mathrm{SMD}$} & $M_{0}(\mathrm{~mL} / \mathrm{gVS})$ & $290.4 \pm 1.3$ & $265.2 \pm 1.3$ & $247.9 \pm 2.3$ & $209.2 \pm 1.5$ & $204.2 \pm 7.5$ \\
& $R_{\mathrm{m}}(\mathrm{mL} / \mathrm{gVS} \cdot \mathrm{d})$ & $39.5 \pm 5.8$ & $36.1 \pm 3.5$ & $33.9 \pm 6.8$ & $33.6 \pm 5.4$ & $22.2 \pm 2.4$ \\
& $k^{\prime}(\mathrm{d})$ & $0.39 \pm 0.11$ & $0.41 \pm 0.10$ & $3.45 \pm 0.15$ & $4.99 \pm 0.12$ & $4.81 \pm 0.12$ \\
\hline \multirow{3}{*}{$\mathrm{STZ}$} & $M_{0}(\mathrm{~mL} / \mathrm{gVS})$ & $293.2 \pm 0.5$ & $259.7 \pm 0.9$ & $248.5 \pm 1.2$ & $218.9 \pm 1.2$ & $139.6 \pm 0.9$ \\
& $R_{\mathrm{m}}(\mathrm{mL} / \mathrm{gVS} \cdot \mathrm{d})$ & $41.8 \pm 2.9$ & $40.1 \pm 1.4$ & $33.1 \pm 1.2$ & $31.3 \pm 1.3$ & $15.8 \pm 0.6$ \\
& $k^{\prime}(\mathrm{d})$ & $0.36 \pm 0.13$ & $1.27 \pm 0.06$ & $1.67 \pm 0.09$ & $1.40 \pm 0.10$ & $1.96 \pm 0.03$ \\
\hline
\end{tabular}

ditions [34]. On the other hand, microorganisms in anaerobic system had high tolerance to these two antibiotics.

\subsubsection{Biochemical methane potential}

Total methane production was obtained by measuring the average methane production of each group (Fig. S2). The proportion of methane in biogas was basically between $65.8 \%$ and $72.5 \%$, and there was no significant change when SAs were added. Meanwhile, the proportion of methane did not change regularly with the concentration of SAs. The results of this experiment were consistent with previous research findings [35, 36]. The methane yield was simulated according to the modified Gompertz equation of Eq. (1), and the modified Gompertz growth curve was shown in Fig. S2, and the estimated kinetic constants were listed in Table 1 . The results 
indicated that all the test groups followed the modified Gompertz growth curve with $R^{2}$ of 0.976-0.997.

When there were no SAs in the anaerobic digestion system, the $M_{0}$ was range from $290.4 \mathrm{~mL} / \mathrm{gVS}$ to $293.2 \mathrm{~mL} / \mathrm{gVS}$, which was similar to those in previous literatures [37]. Adding SAs in anaerobic digestion system, $M_{0}$ and $R_{\mathrm{m}}$ were inhibited to varying degrees, and $k^{\prime}$ was prolonged. The concentration of SAs remained relatively low, the $M_{0}$ was inhibited by $14.8 \%$ to $27.9 \%$. At the same time, the $R_{\mathrm{m}}$ can also be maintained at about $90 \mathrm{~mL} / \mathrm{gVS} \cdot \mathrm{d}$. This indicated that the operation of anaerobic digestion system was basically normal $[36,38]$. The concentration of SAs reached a certain height, the $M_{0}$ and $R_{m}$ would decrease sharply. When the concentration of SQX and STZ reached $100 \mathrm{mg} / \mathrm{L}$ in anaerobic digestion system, the $M_{0}$ was $149.9 \mathrm{~mL} / \mathrm{gVS}$ and $139.6 \mathrm{~mL} / \mathrm{gVS}$, respectively, which was $48.9 \%$ and $52.2 \%$ lower than that of control group. The results showed that the addition of high concentration of SAs seriously inhibited the stability of a certain process of methane production in anaerobic digestion system. Moreover, microorganisms need a certain time to adapt to the environment where SAs exist, resulting in the $R_{m}$ far below the normal level [39].
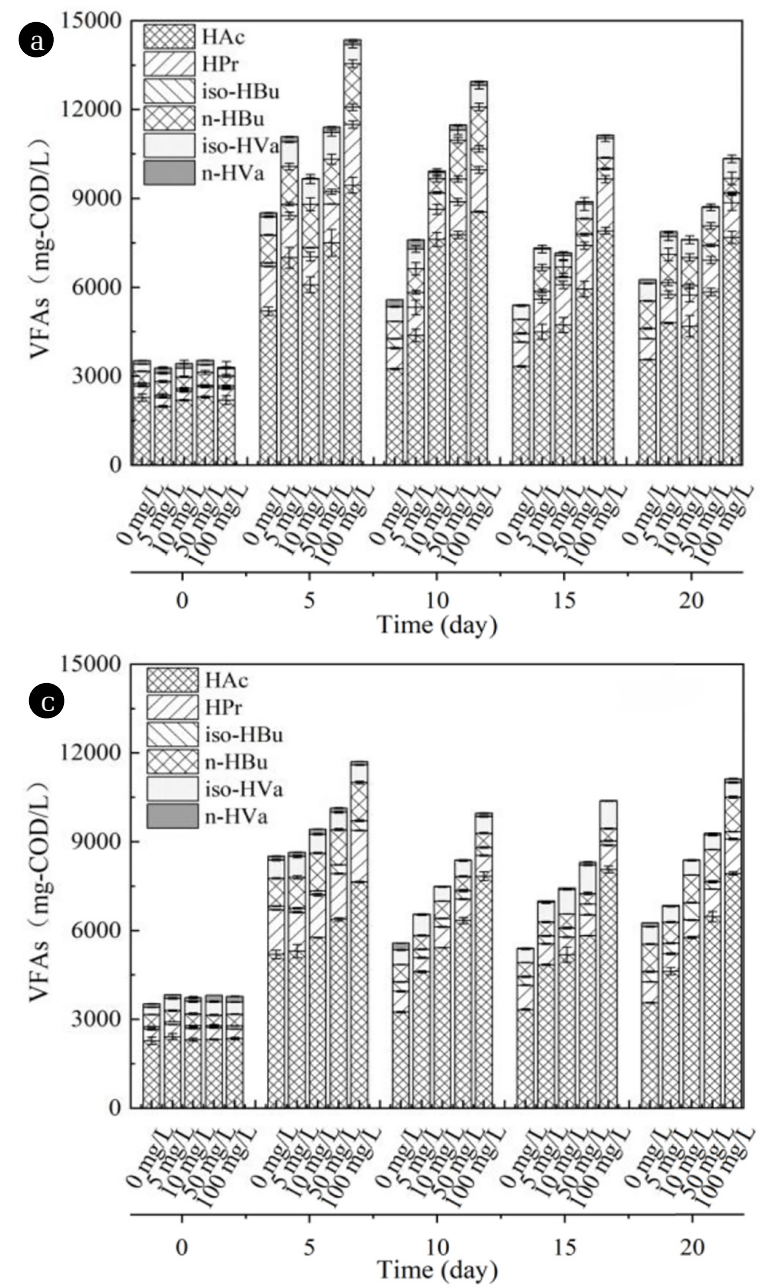

Fig. 2. Effect of SAs on variation of VFAs concentrations. (a)-(d) showed the effects of (a) SQX, (b) SMX, (c) SMD and 9d) STZ on VFAs concentrations in anaerobic digestion.

Among them, methane production rate and methane yield were very important indicators for large-scale biogas plants. In order to reduce the residence time of substrates, rapid and efficient degradation of substrates was also one of the important indicators to be considered. $k$ can well characterize the time required for substrate degradation. $k$ ' was prolonged with the increasing concentration of SAs. When the concentration of SAs in the substrate of biogas plant exceeded a certain stage, the biogas production would be further reduced without increasing the residence time.

\subsection{Effect of SAs on Anaerobic Digestion Performance}

\subsubsection{Volatile fatty acids}

The content of VFAs increased sharply at first and then slowly decreased to an equilibrium value with the digestion proceeding (Fig. 2). Of all the VFAs, HAc accounted for the largest proportion, and was one of the components with the greatest change in content. In addition, $\mathrm{HPr}$, iso- $\mathrm{HBu}$ and $\mathrm{n}-\mathrm{HBu}$ also accumulated in varying degrees. The SAs had little effect on the production of VFAs at low concentrations. Because methane can be decomposed and trans-
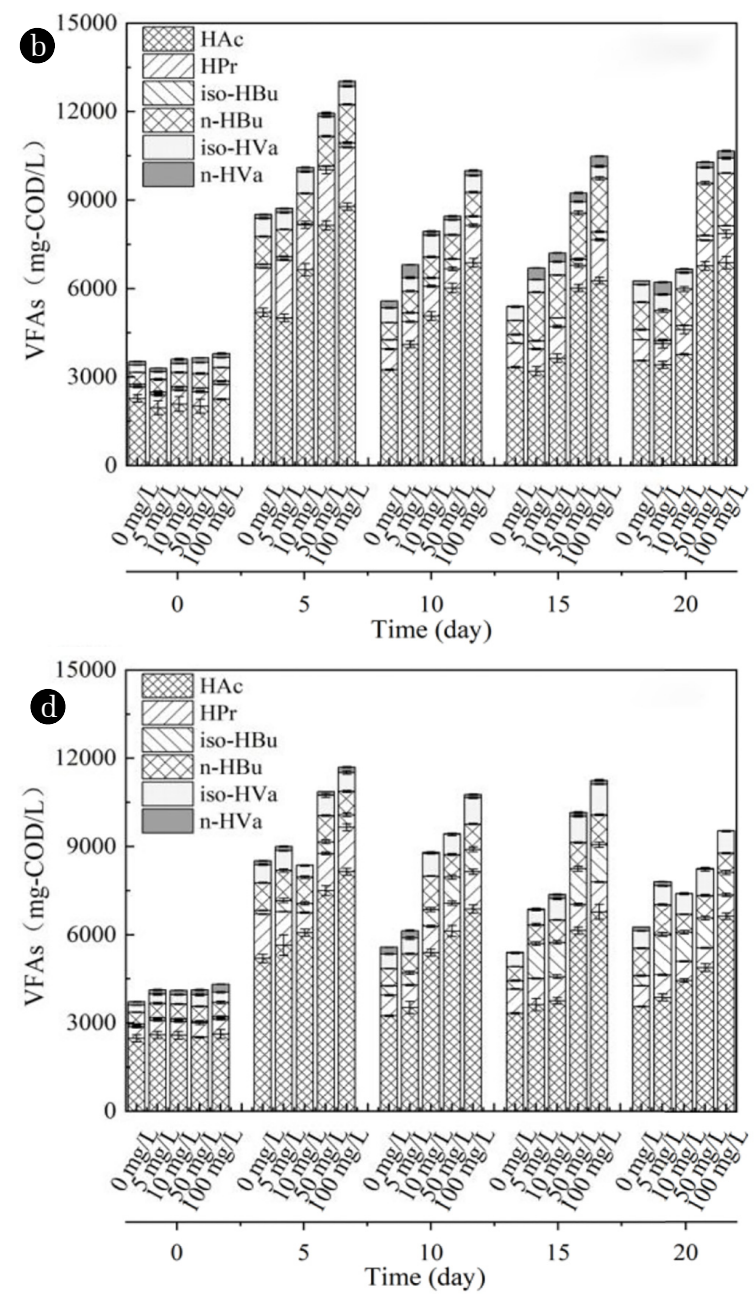
formed from VFAs, excessive accumulation of VFAs had an important impact on the performance of anaerobic bacteria in the reactor [40]. Therefore, SAs with low concentration had little effect on microorganisms in anaerobic digestion system. When the concentration of SAs exceeded $50 \mathrm{mg} / \mathrm{L}$, the concentration of HAc was raised sharply. Among them, when the concentration of SQX were $50 \mathrm{mg} / \mathrm{L}$ and $100 \mathrm{mg} / \mathrm{L}$, the concentration of HAc was 1.4 and 1.8 times higher than that in the absence of SQX, respectively.

With the progress of anaerobic digestion, the content of VFAs did not fall to the normal level, which would have a negative impact on the stable operation of the system [41]. The disparity was unobserved on the production of $\mathrm{HPr}$, iso- $\mathrm{HBu}, \mathrm{n}-\mathrm{HBu}$, iso- $\mathrm{HVu}$ and n-Hvu among these reactors with different SMX, SMD and STZ dosages. With the stable operation of anaerobic digestion, the concentration of HAc varied significantly while SAs were added. HAc was increased from 3243-3559 $\mathrm{mgCOD} / \mathrm{L}$ to $7833-7924$ mgCOD/L while the SMX dosage increased from 0 to $100 \mathrm{mg} / \mathrm{L}$. Similarly, with the SMD and STZ dosage increased from 0 to 100 $\mathrm{mg} / \mathrm{L}$, the final accumulation of HAc reached $6875 \mathrm{mgCOD} / \mathrm{L}$ and $6638 \mathrm{mgCOD} / \mathrm{L}$. To further determine the cause of HAc accumulation, the amount of HAc accumulation was compared with the amount of methane inhibition, which was based on the transformation factor (0.35 $\mathrm{L} \mathrm{CH}_{4} / \mathrm{g} \mathrm{COD)} \mathrm{[42,} \mathrm{43].} \mathrm{The} \mathrm{addition} \mathrm{of} \mathrm{SQX}$ was mainly due to the inhibition of the methanogenesis process, which leaded to the accumulation of HAc. The effect of SMX on the accumulation of HAc and the methanogenesis process was not obvious, but the slight increase in the content of HAc was also related to the inhibitory effect on the methanogenesis process. The addition of $100 \mathrm{mg} / \mathrm{L}$ of SMD resulted in the accumulation of HAc, $62 \%$ of which was caused by the inhibition of the methanogenesis process, and the remaining HAc accumulation was due to the promotion of the hydrolysis and acidification process. The addition of STZ not only inhibited the methanogenesis process, but also had a certain inhibitory effect on the hydrolysis and acidification process, but the inhibitory effect on the methanogenesis was more obvious.

\subsubsection{Total solid}

Swine manure contains a lot of fat, protein and carbohydrates, most of which exist as solids in substrates. SAs reduced the degradation of organic matter in swine manure by inhibiting microbial activity in the environment (Fig. 3). The gradual increase in TS removal rate was associated with the growth of methanogenic microorganisms over the initial period of time. The addition of SAs inhibited the average removal rate of TS, which was related to the decrease of biomass concentration represented by previous studies by Shanmugam [44]. When the concentration of SQX reached $100 \mathrm{mg} / \mathrm{L}$, the inhibition of TS degradation rate was most obvious. On the fifth day of experiment, the degradation rate of TS decreased from $10.5 \%$ to $8.1 \%$, which was much lower than
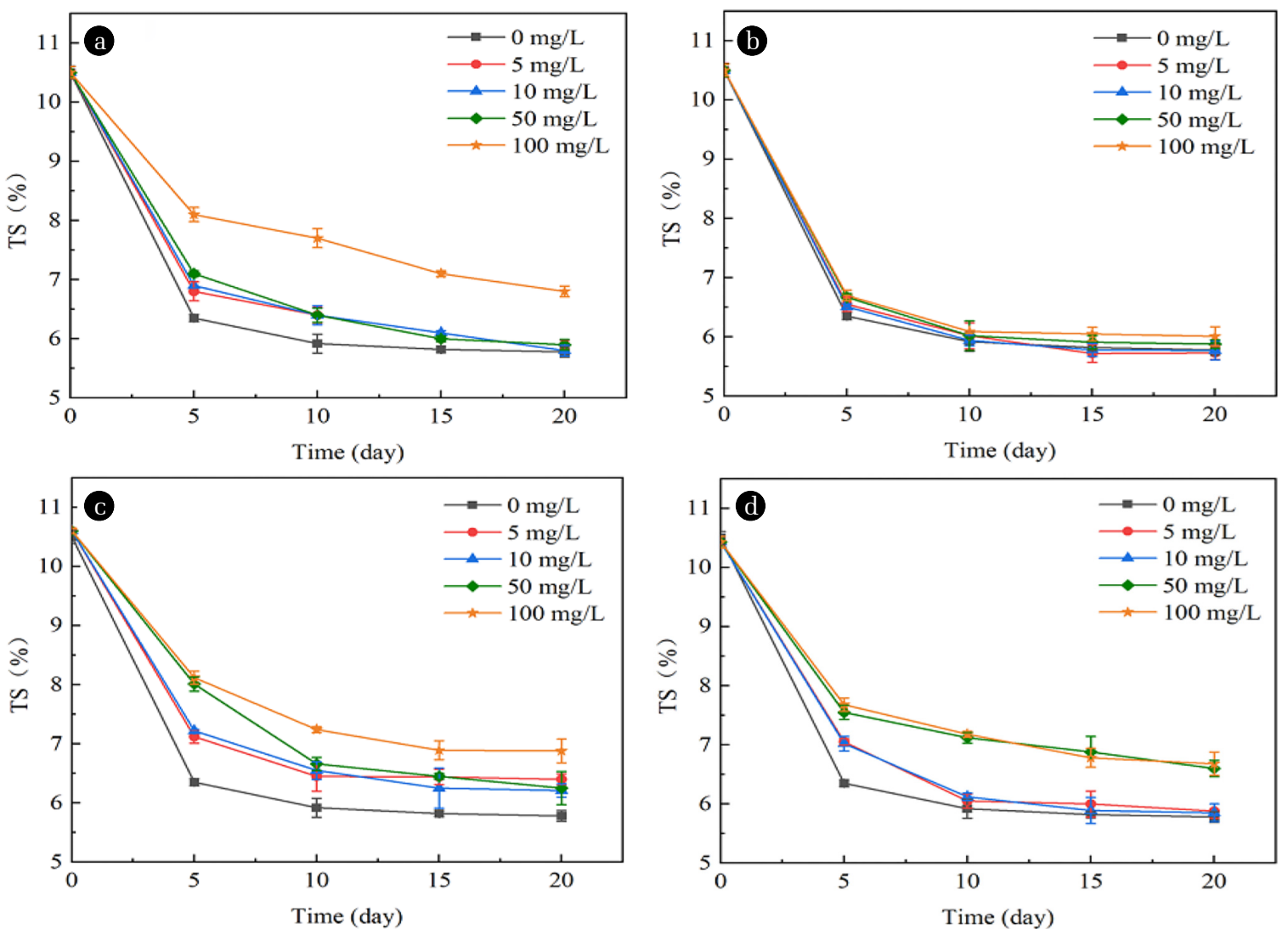

Fig. 3. Effect of SAs on the removal of TS. (a)-(d) showed the effects of (a) SQX, (b) SMX, (c) SMD and (d) STZ on the removal of TS in anaerobic digestion. 
that of $6.4 \%$ without adding SAs. The addition of SMX had no effect on the change of TS, indicating that SMX had no effect on the microorganisms degrading macromolecular solid organic matter. With the increase of SMD concentration, the degradation efficiency of TS decreased gradually. However, when high concentrations of SAs are added, the degradation efficiency of TS changed greatly. It is suggested that high concentration of SAs inhibited the degradation rate of degradable organic compounds. In addition, SAs also inhibited the degradation rate of easily degradable organic matter [45], resulting in the reduction of methane production efficiency.

\subsubsection{Total organic carbon}

The change of TOC was contrary to the degradation efficiency of substrate in digestion system (Fig. 4). Combined with the degradation rate of TS, the main speed-limiting steps of SAs for biogas production can be found by analyzing the conversion rate of TOC to $\mathrm{CH}_{4}$ and $\mathrm{CO}_{2}$ in the system. Methanosarcina barkeri have shown that approximately $95 \%$ of the degradable organic matter was converted to biogas, $3 \%$ was converted to cell material and the remaining $2 \%$ of the substrate was needed for energy supply [46]. The TOC content reflected the conversion rate of carbon element into $\mathrm{CH}_{4}$ and $\mathrm{CO}_{2}$ in biogas slurry. When a lower concentration of SAs was added, the biogas yield, TS and TOC did not change significantly with the control group. Combining biogas yield and TS degradation efficiency analysis, low concentration of SAs did not affect the activity of microorganisms in methane production process. When the concentration of SAs increased, the inhibitory effect of SAs was different. SQX inhibited microorganisms that degrade macromolecule solid organic matter, but had little effect on the conversion of soluble organic matter into biogas. The addition of SMX had no effect on the degradation of organic matter and the conversion of organic matter to biogas in the system. This may be due to the degradation of SMX by microorganisms, and the toxicity of its degradation products to microorganisms was not obvious [47]. The degradation efficiency of TOC by adding SMD and STZ in anaerobic digestion system was similar, and decreased with the increase of SAs concentration. However, STZ had no effect on the degradation of TS at low concentration, which indicated that STZ at low concentration did not affect the degradation of macromolecule solid organic matter, but affected the degradation of dissolved organic matter.

\subsection{Effect of SAs on Microbial Community}

For bacterial communities analysis, 53,716-69,185 clean reads were obtained for each sample with an average length of $200 \mathrm{bp}$ after removing low quality sequences and chimeras. The sequence number of each sample was normalized and 941-1102 operational taxonomic units (OTUs) were generated. Analysis of archaeal commun-
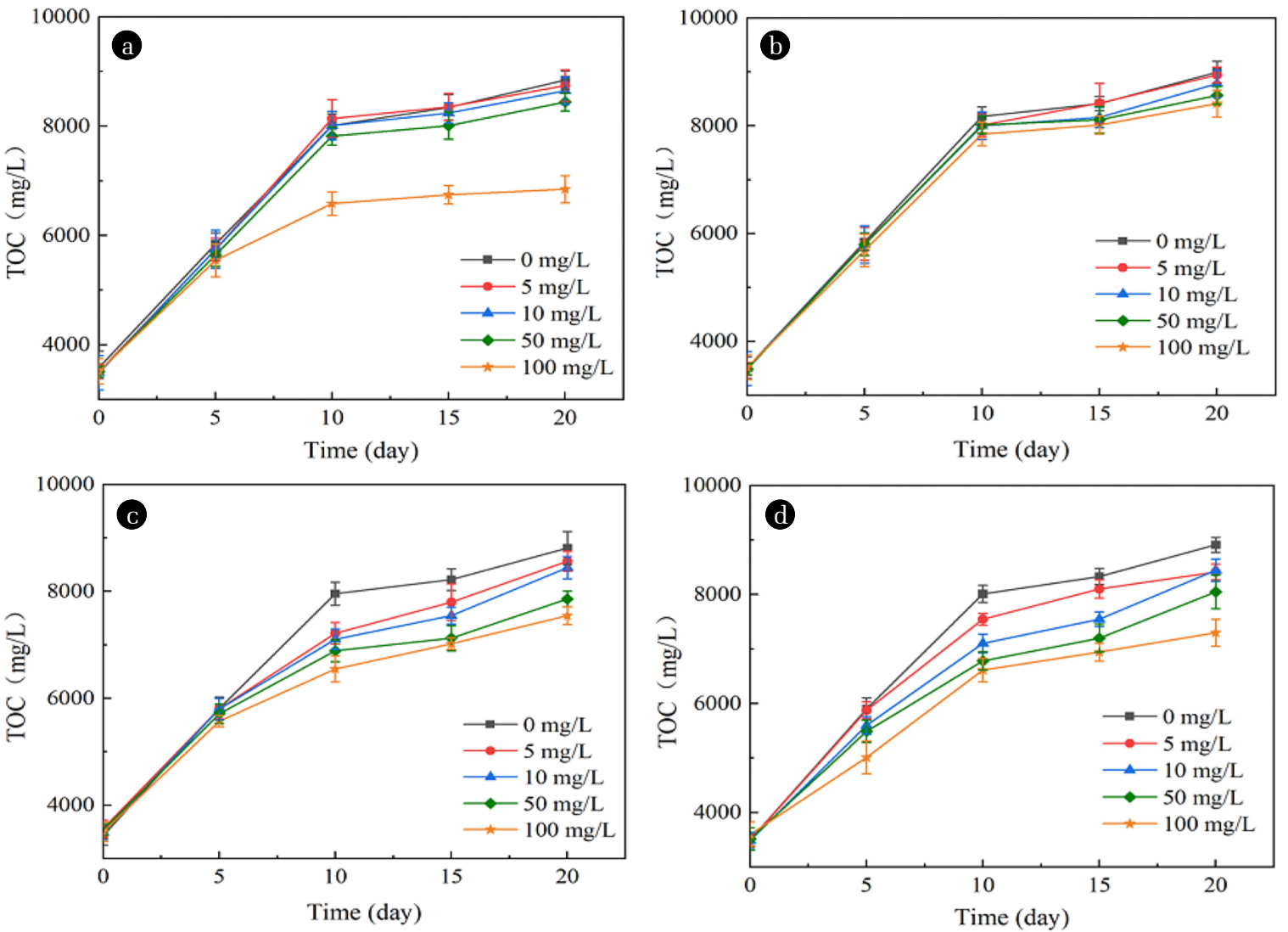

Fig. 4. Effect of SAs on the change of TOC. (a)-(d) showed the effects of (aO SQX, (b) SMX, 9c) SMD and (d) STZ on the change of TOC in anaerobic digestion. 
ities resulted in 48,306-58,577 sequences for each sample with an average length of $200 \mathrm{bp}$. Richness and diversity of the bacterial and archaeal communities were indicated by OUTs, Chao 1 value, ACE index and Shannon index (Table S2).

For bacterial communities, the number of OTUs decreased after SAs were added to the anaerobic digestive system, especially SQX. The Shannon index of the high concentration SQX group decreased significantly from $3.81 \pm 0.02$ to $3.57 \pm 0.16$, while that of the other SAs groups decreased from 3.68-3.75 $(\mathrm{P}<0.05)$. The Chao1 index values in the experimental groups with adding SAs showed no showed significant difference $(\mathrm{P}>0.05)$, but all were lower than the original sludge in groups without SAs $(\mathrm{P}<0.05)$. Adding high concentration SAs in anaerobic system could significantly reduce the diversity and richness of microbial community in the environment. Towards the archaeal community, the richness of all groups was reduced, which was reflected in the decrease in the number of OTUs, Chao 1 and ACE (Table S2). This also revealed that the high concentration of SAs negatively affected the richness of the archaeal community. Moreover, high concentration SAs only reduced the archaeal richness rather than their diversity during the anaerobic digestion process. Meanwhile, nonmetric multidimensional scaling (NMDS) was carried out to analyze the differences of microbial communities of all samples (Fig. S3). In the MDS-map, space and distance were used to represent the relationship between samples. After adding SAs, the microbial community structure including bacteria and archaea in the system was similar. This was closely related to para-amino benzene sulfonamide, a special structure of SAs, which could inhibit the synthesis of folic acid required for synthesis of purines and nucleic acid. The microbial community structure of the anaerobic digestive system with adding SAs was significantly different from that of the blank control group. In the bacterial community, SQX had a greater impact on the bacterial community structure, followed by STZ and SMD, and SMX had the smallest impact. Among archaea communities, SQX and STZ had little effect on the structure of archaea community compared with the blank group, SMD and SMX had greater effect on the structure of archaea community.

The taxonomic compositions of the microbial community at phyla level were shown in Fig. S4. Of the total sequences, less than $1 \%$ were not classified at any phylum level. At phylum level, Firmicutes (61.0\%), Bacteroidetes (19.7\%) and Proteobacteria (15.3\%) were the primary phylum in the blank group. The addition of SAs resulted in the decrease of Proteabacteria, compared with the blank group. With the addition of SQX, the relative abundance of Bacteroidetes increased, while the relative abundance of Bacteroidetes decreased with the addition of other SAs. In order to further reveal the bacteria shift under high concentration SAs inhibition, the phylogenetic identities of different genera was illustrated (Fig. 5). The general level changes of bacterial community in the blank group and after adding SAs were obtained. At the genus level, Clostridium III, Mobilitalea, Bacteroides and Ignatzschineria were the primary genera in the system. After adding SAs, the relative abundance of Clostridium III increased. Clostridium III species, which belong to the Firmicutes phylum, was frequently observed in the anaerobic systems. Clostridium III degraded sugar and protein into small molecular substances and organic acids, which was related to the increase of VFAs content caused by the

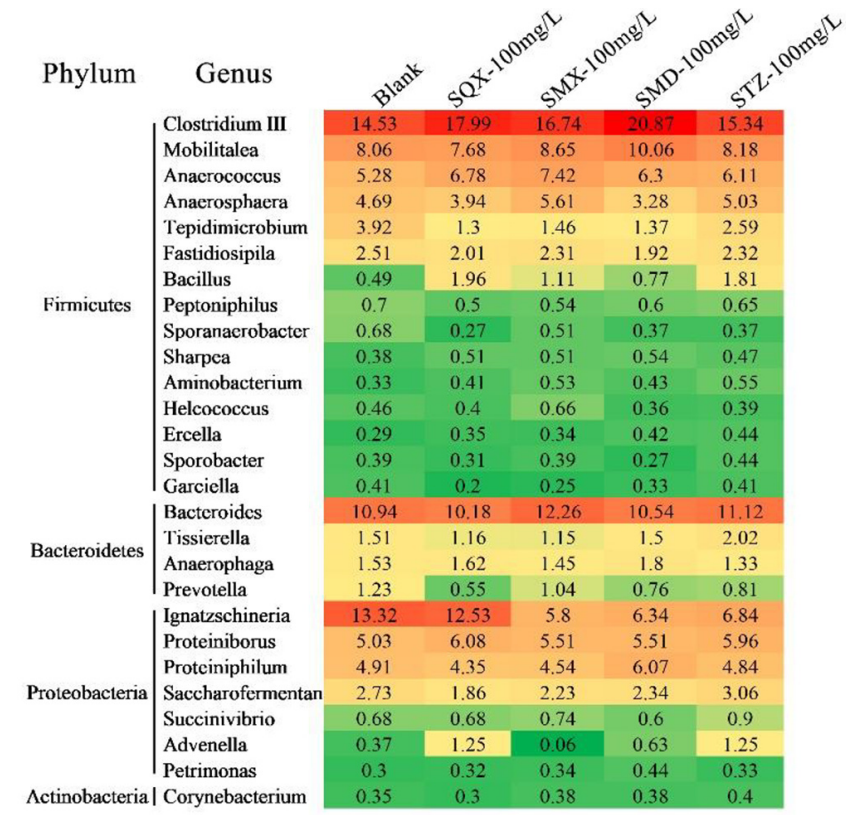

Fig. 5. The phylogenetic identities of different genera.

addition of SAs in front. The results of this study are consistent with those of previous studies [48]. Propionate-degrading bacteria and butyrate-degrading bacteria were not strictly arranged according to a single class, family or order, even not phylum [49]. SAs sometimes did not completely limit the degradation of $\mathrm{HPr}$, iso- $\mathrm{Hbu}$, $\mathrm{n}-\mathrm{HBu}$ and valeric acid, because antibiotics did not completely inhibit all bacteria that degraded $\mathrm{HPr}$ or iso- $\mathrm{HBu}$ and $\mathrm{n}-\mathrm{HBu}$ [50]. Therefore, HPr, iso-HBu and n-HBu did not accumulate in large quantities when some SAs were present in the anaerobic digestion system. In addition, only HAc was affected by SAs in the experimental results of VFAs. This revealed that SMD and STZ had effects on the relative abundance of Clostridium III, but had similar effects on the content of VFAs, especially HAc. Mobilitalea, a novel strictly anaerobic, could degrade glucose into acetate, ethanol, $\mathrm{H}_{2}$ and $\mathrm{CO}_{2}$. Therefore, the increase of HAc concentration caused by the addition of SMD may be due to the increase of Mobilitalea relative abundance. Ignatzschineria, as a common microorganism in anaerobic digestion system, belonged to the Proteobacteria phylum which can degrade macromolecule organic matter. When high concentrations of SAs were added, the relative abundance of Ignatzschineria decreased significantly. These results indicate that SAs inhibited the growth and reproduction of Ignatzschineria, and reduced the degradation efficiency of TS.

There were only four major genera, Methanomassiliicoccus, Methanobrevibacter, Methanoculleus and Methanosphaera, which were predominant in all groups and accounted for more than $98.4 \%$ of all archaea (Table 2). The genus Methanomassiliicoccus was extraordinary dominant in the blank group (74.2\%), but significantly reduced to $61.1 \%-66.3 \%$ when SAs with high concentration were added during the anaerobic digestion process. In addition, this genus showed more dominant in the group with adding SMD, which sequence percentage was $66.3 \%$. Comparatively, the genus Methanobrevibacter, which was the second primary genera in the blank group (25.2\%), increased enormous reaching 37.3\%-38.0\% 
Table 2. The Relative Abundances of the Archaeal Community at Genus Level

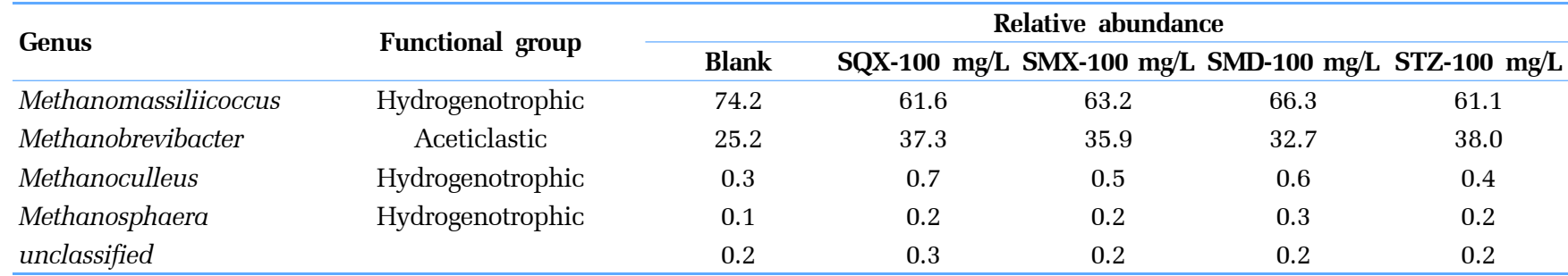

in the group with adding SQX, SMX and STZ, respectively. According to their functional classification, Methanomassiliicoccus, Methanoculleus and Methanosphaera belonged to hydrogenotrophic, while Methanobrevibacter belonged to aceticlastic. It was worth noting that all the major hydrogenotrophic methanogens showed a significantly decrease in response to adding high concentration SAs, while the aceticlastic methanogens increased. This may be due to the adaptation and migration of methanogenic bacteria, that is to say, the ccumulation of VFAs could promote the transformation of methanogenic bacteria into hydrogenotrophic methanogenic bacteria [51]. Increasing the relative abundance of aceticlastic methanogens accelerated the degradation of organic matter into VFAs in anaerobic digestion system, which resulted in the accumulation of VFAs. However, excessive VFAs in turn inhibited the growth of methanogens and reduced methane production. Similarly, Merlino et al. [52] speculated that with the partial accumulation of VFAs, especially HAc and HPr, the proportions of methanogens and the acetotrophic methanogen decreased by an order of magnitude.

It is noteworthy that archaea was considered to be extensively resistant to antibiotics, but even closely related species were susceptible to different antibiotics [53]. Unlike bacteria, the cell wall characteristics of archaea were not potential targets for antibiotics [54]. In addition, the same antibiotic had multiple targets or require specific targets. Therefore, the effects of SAs on bacteria and archaea were different. The addition of SAs in the anaerobic digestive system can affected the electronic transport system, making it difficult for microorganisms to absorb nutrients, especially anaerobic archaea [55]. Compared with the diversity of bacterial, the richness of archaeal population decreased significantly. Therefore, it is more convenient and direct to study the effect of SAs on archaea than bacteria.

\subsection{Correlation between Environmental Factors and Microbial Community Structure}

Fig. 6 showed the relationship between the dominant bacteria, archaea and environmental factors in anaerobic digestion system after adding SAs. Without adding SAs, the total methane production was positively correlated with TS removal rate, TOC concentration and relative abundance of Methanomassiliicoccus in anaerobic digestion system. The accumulation of VFAs was negatively correlated with total methane production, and HAc was dominant.

The addition of antibiotics had different effects on microbial community structure and environmental factors in the system. The concentration changes of iso-HVa, n-HVa, HPr and HAc were related to the addition of high concentration SQX in the system.

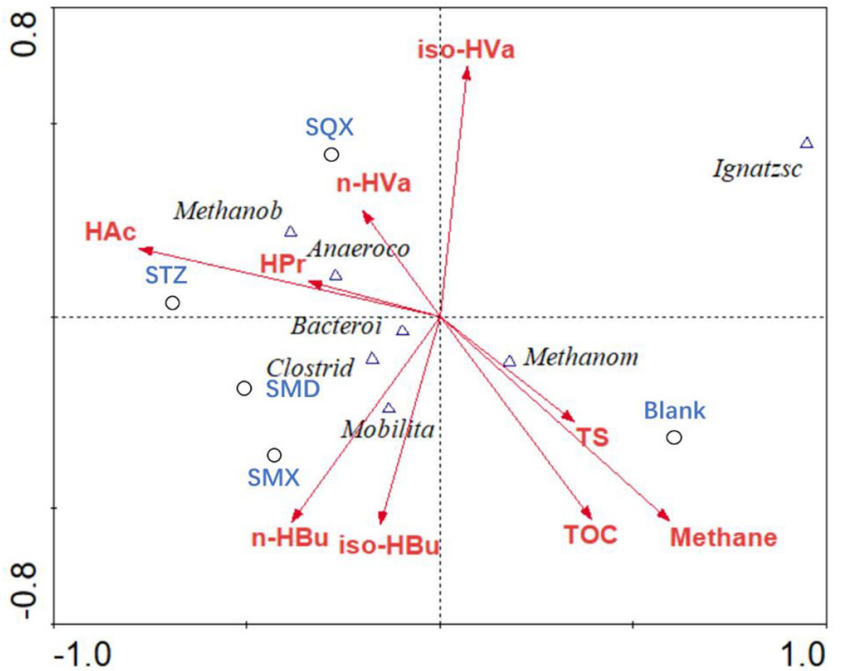

* The change of microorganism is represented by the change of its relative abundance

Fig. 6. Canonical Correspondence Analysis (CCA) of shifts in the quantitative structure of bacterial and archaeal community* and their correlation between species of VFAs, TOC, TS and accumulated methane.

At the same time, adding high concentration SQX had a positive correlation with the relative abundance of Methanobrevibacter and Anearococcus, and led to the increase of the content of VFAs. High concentration of SQX showed the strongest negative correlation with total methane production, this corresponded to the experimental results. Compared with SQX, high concentration of STZ showed a higher positive correlation with HAc and HPr, and had a stronger impact on HAc. In addition, STZ also showed positive correlation with many bacteria, such as Anaerococcus, Clostridium III and Bacteroides, but their correlation was not strong. The influence of SMD on microorganisms and other environmental factors in the system was higher than that of SMX. They were positively correlated with the relative abundance of Bacteroides, Clostridium III and Mobilitalea. SMD was the most correlated with the relative abundance of Clostridium III and SMX was the most correlated with the relative abundance of Mobilitalea. Therefore, the degradation efficiency of organic matter in substrates with adding SMX was higher than that with adding SMD. It is noteworthy that high concentrations of SMD and SMX were negatively correlated with the relative abundance of Methanomassiliicoccus in archaea, resulting in the same effects of SMD and SMX addition on the methano- 
genesis stage of microorganisms.

The effects of Bacteroides, Clostridium III, Anaerococcus, Mobilitalea and Methanomassiliicoccus on the whole anaerobic digestion system are not very strong [56]. The relative abundance of Ignatzschineria did not seem to be positively correlated with the presence of other bacteria, but adding SAs had an important influence in Ignatzschineria. However, the change of Ignatzschineria relative abundance had no effect on methane production, because the angle between the point of Ignatzschineria and the line of methane production was nearly 90 degrees.

\section{Conclusions}

Low concentration of SAs had little effect on the stable operation of anaerobic digestion system, which was mainly manifested in the accumulation of VFAs and the degradation rate of TS. When the concentration of SAs reached a certain threshold, it had an irreversible impact on the anaerobic digestive system, resulting in a sharp decline in methane production. The addition of SQX mainly reduced the relative abundance of Methanobrevibacter and resulted in the accumulation of VFAs, especially HAc, which inhibited the production of methane. The addition of SMD and STZ inhibited the growth and reproduction of Proteobacteria, resulting in the degradation of solid organic matter in the substrate. However, the presence of SMD increased the relative abundance of Methanomassiliicoccus, so the inhibition rate of STZ on methane production was higher than that of SMD. The effects of SMX on microbial community structure and influencing factors in the system were smaller than other SAs, so methane production did not decrease significantly. The results provide theoretical support for the effects of SAs with different concentrations on anaerobic digestibility of swine manure.

\section{Acknowledgment}

This research was financially supported by the National Science and Technology Major Projects Special for Water Pollution Control and Management (No. 2017ZX07107-005). The authors declare they have no conflict of interest.

\section{Author Contributions}

S.W. (Ph.D. student) conducted all the experiments and wrote the manuscript. K.D. (Ph.D.), H.C. (Associate Professor) and F.W. (Professor) revised the manuscript. R.Y. (Associate Professor) and B.Z. (Professor) wrote and revised the manuscript.

\section{References}

1. Sharpe RR, Harper LA, Byers FM. Methane emissions from swine lagoons in Southeastern US. Agr. Ecosyst. Environ. 2002;90:17-24

2. Lesteur M, Bellon-Maurel V, Gonzalez C, Latrille E. Roger JM,
Junqua G. Alternative methods for determining anaerobic biodegradability: A review. Process Biochem. 2010;45:431-440.

3. Chiu SLH, Lo IMC. Reviewing the anaerobic digestion and co-digestion process of food waste from the perspectives on biogas production performance and environmental impacts. Environ. Sci. Pollut. R. 2016;23:24435-24450.

4. Cetecioglu Z, Ince B, Gros M, Rodriguez-Mozaz S, Barcelo D, Ince O. Biodegradation and reversible inhibitory impact of sulfamethoxazole on the utilization of volatile fatty acids during anaerobic treatment of pharmaceutical industry wastewater. Sci. Total Environ. 2015;536:667-674.

5. Bialk-Bielinska A, Caban M, Pieczynska A, Stepnowski P, Stolte S. Mixture toxicity of six sulfonamides and their two transformation products to green algae Scenedesmus vacuolatus and duckweed Lemna minor. Chemosphere 2017;173:542-550.

6. Zhao L, Dong YH, Wang H. Residues of veterinary antibiotics in manures from feedlot livestock in eight provinces of China. Sci. Total Environ. 2010;408:1069-1075.

7. Xianguang H, Yi L, Qixing Z, Lin X. Determination of thirteen antibiotics residues in manure by solid phase extraction and high performance liquid chromatography. Chinese J. Anal. Chem. 2008;36:1162-1166.

8. Martinez-Carballo E, Gonzalez-Barreiro C, Scharf S, Gans O. Environmental monitoring study of selected veterinary antibiotics in animal manure and soils in Austria. Environ. pollut. 2007;148:570-579.

9. Narihiro T, Sekiguchi Y. Microbial communities in anaerobic digestion processes for waste and wastewater treatment: A microbiological update. Curr. Opin. Biotech. 2007;18:273-278.

10. Ince B, Coban H, Turker G, Ertekin E, Ince O. Effect of oxytetracycline on biogas production and active microbial populations during batch anaerobic digestion of cow manure. Bioproc. Biosyst. Eng. 2012;36:541-546.

11. Arikan OA, Sikora LJ, Mulbry W, Khan SU, Rice C, Foster GD. The fate and effect of oxytetracycline during the anaerobic digestion of manure from therapeutically treated calves. Process Biochem. 2006;41:1637-1643.

12. Shi JC, Liao XD, Wu YB, Liang JB. Effect of antibiotics on methane arising from anaerobic digestion of pig manure. Anim. Feed Sci. Tech. 2011;166:457-463.

13. Loftin KA, Henny C, Adams CD, Surampali R, Mormile MR. Inhibition of microbial metabolism in anaerobic lagoons by selected sulfonamides tetracyclines lincomycin and tylosin tartrate. Environ. Toxicol. Chem. 2005;24:782-788.

14. Aydin S, Ince B, Ince O. Application of real-time PCR to determination of combined effect of antibiotics on Bacteria Methanogenic Archaea Archaea in anaerobic sequencing batch reactors. Water Res. 2015;76:88-98.

15. Hu J, Xu Q, Li X, et al. Sulfamethazine (SMZ) affects fermentative short-chain fatty acids production from waste activated sludge. Sci. Total Environ. 2018;639:1471-1479.

16. Oh YK, Park CH, Han GB. Enhancement of anaerobic co-digestion in acidogenic (ACIDO-DR) and methanogenic (METHA-DR) digester system. J. Taiwan Ins. Chem. E. 2017;72:149-156.

17. Mai, DT, Stuckey DC, Oh S. Effect of ciprofloxacin on methane production and anaerobic microbial community. Bioresour. Technol. 2018;261:240-248. 
18. Kim S, Bae J, Choi O, et al. A pilot scale two-stage anaerobic digester treating food waste leachate (FWL): Performance and microbial structure analysis using pyrosequencing. Process. Biochem. 2014;49:301-308.

19. Leaphart AB, Lovell CR. Recovery and analysis of formyltetrahydrofolate synthetase gene sequences from natural populations of acetogenic bacteria. Appl. Environ. Microb. 2001;67:1392-1395.

20. Aydin S, Ince B, Ince O. Inhibitory effect of erythromycin tetracycline and sulfamethoxazole antibiotics on anaerobic treatment of a pharmaceutical wastewater. Water Sci. Tech. 2015;71: 1620-1628.

21. Achinas S, Li Y, Achinas V, Willem Euverink GJ. Influence of sheep manure addition on biogas potential and methanogenic communities during cow dung digestion under mesophilic conditions. Sustain. Environ. Res. 2018;28:240-246.

22. Bauer A, Lizasoain J, Nettmann E, et al. Effects of the antibiotics chlortetracycline and enrofloxacin on the anaerobic digestion in continuous experiments. Bioenerg. Res. 2014;7:1244-1252.

23. Blackwell PA, Holten Lutzhoft HC, Ma HP, Halling-Sorensen B, Boxall AB, Kay P. Ultrasonic extraction of veterinary antibiotics from soils and pig slurry with SPE clean-up and LC-UV and fluorescence detection. Talanta 2004,64:1058-1064.

24. Hoff R, Pizzolato TM, Diaz-Cruz MS. Trends in sulfonamides and their by-products analysis in environmental samples using mass spectrometry techniques. Trends Environ. Anal. 2016;9: 24-36.

25. Gilcreas FW. Standard methods for the examination of water and waste water. Am. J Public Health. 1966;56:387-388.

26. Li C, Champagne P, Anderson BC. Evaluating and modeling biogas production from municipal fat oil and grease and synthetic kitchen waste in anaerobic co-digestions. Bioresour. Technol. 2011;102:9471-9480.

27. Takahashi S, Tomita J, Nishioka K, Hisada T, Nishijima M. Development of a prokaryotic universal primer for simultaneous analysis of bacteria and archaea using next-generation sequencing. PLoS One 2014; 9:105592-110601.

28. Takai K, Horikshi K. Rapid detection and quantification of members of the Archaeal community by quantitative PCR using fluorogenic probes. Appl. Environ. Microb. 2000;66:5066-5072.

29. Van Epps A, Blaney L. Antibiotic residues in animal waste: Occurrence and degradation in conventional agricultural waste management practices. Curr. Pollut. Rep. 2016,2:135-155.

30. Miran W, Jang J, Nawaz M, Shahzad A, Lee DS. Biodegradation of the sulfonamide antibiotic sulfamethoxazole by sulfamethoxazole acclimatized cultures in microbial fuel cells. Sci. Total Environ. 2018;627:1058-1065.

31. Chen Y, Wei Z, Ying M, et al. Effects of SDM Concentration on Methane Production and Enzyme Activities during Anaerobic Fermentation. Environ. Sci. Tech. 2017;40:18-24.

32. Garrett ER, Wright OK. Kinetics and mechanisms of action of drugs on microorganisms VII-Quantitative adherence of sulfonamide action on microbial growth to a receptor. J Pharm. Sci. 1967;56:1576-1585.

33. Mondal S, Mandal SM, Mondal T.K, Sinha C. Structural characterization of new Schiff bases of sulfamethoxazole and sulfathiazole their antibacterial activity and docking computation with
DHPS protein structure. Spectrochim. ACTA A. 2015;150: 268-279.

34. Jia Y, Khanal SK, Zhang H, Chen GH, Lu H. Sulfamethoxazole degradation in anaerobic sulfate-reducing bacteria sludge system. Water Res. 2017;119:12-20.

35. Spielmeyer A, Breier B, Groissmeier K, Hamscher G. Elimination patterns of worldwide used sulfonamides and tetracyclines during anaerobic fermentation. Bioresour. Technol. 2015;193: 307-314.

36. Li L, Wang R, Jiang Z, Li W, Liu G, Chen C. Anaerobic digestion of tobacco stalk: biomethane production performance and kinetic analysis. Environ. Sci. Pollut. Res. 2019;26:14250-14258.

37. Kafle GK, Chen L. Comparison on batch anaerobic digestion of five different livestock manures and prediction of biochemical methane potential (BMP) using different statistical models. Waste Manag. 2016;48:492-502.

38. Mao C, Wang X, Xi J, Feng Y, Ren G. Linkage of kinetic parameters with process parameters and operational conditions during anaerobic digestion. Energy 2017;135:352-360.

39. Kafle GK, Sang HK. Anaerobic treatment of apple waste with swine manure for biogas production: Batch and continuous operation. Appl. Energ. 2013;103:61-72.

40. Colmenarejo MF, Sánchez E, Bustos A, García G, Borja R. A pilot-scale study of total volatile fatty acids production by anaerobic fermentation of sewage in fixed-bed and suspended biomass reactors. Process Biochem. 2004;39:1257-1267.

41. Zhang C, Xiao G, Peng L, Su H, Tan T. The anaerobic co-digestion of food waste and cattle manure. Bioresour. Technol. 2013;129:170-176.

42. Kim J, Kim J, Lee C. Anaerobic co-digestion of food waste, human feces, and toilet paper: Methane potential and synergistic effect. Fuel 2019;248:189-195.

43. Li Y, Jin Y, Li J, Li H, Yu Z. Effects of thermal pretreatment on the biomethane yield and hydrolysis rate of kitchen waste. Appl. Energ. 2016;172:47-58.

44. Shanmugam SR, Adhikari S, Nam H, Kar Sajib S. Effect of bio-char on methane generation from glucose and aqueous phase of algae liquefaction using mixed anaerobic cultures. Biomass Bioenerg. 2018;108:479-486.

45. Sponza DT, Demirden P. Treatability of sulfamerazine in sequential upflow anaerobic sludge blanket reactor (UASB)/ completely stirred tank reactor (CSTR) processes. Sep. Purif. Technol. 2007;56:108-117.

46. Kythreotou N, Florides G, Tassou SA. A review of simple to scientific models for anaerobic digestion. Renew. Energ. 2014;71:701-714.

47. Ghattas AK, Fischer F, Wick A, Ternes TA. Anaerobic biodegradation of (emerging) organic contaminants in the aquatic environment. Water Res. 2017;116:268-295.

48. Lins P, Reitschuler C, Illmer P. Methanosarcina spp. the key to relieve the start-up of a thermophilic anaerobic digestion suffering from high acetic acid loads. Bioresour. Technol. 2014;152:347-354.

49. Lins P, Reitschuler C, Illmer P. Impact of several antibiotics and 2-bromoethanesulfonate on the volatile fatty acid degradation methanogenesis and community structure during thermophilic anaerobic digestion. Bioresour. Technol. 2015;190:148-158. 
50. Maleki S, Derikvand M, Dalvand M, Ebrahimi G. Load-carrying capacity of mitered furniture corner joints with dovetail keys under diagonal tension load. Turk. J. Agric. For. 2012;36:636-643.

51. Hori T, Haruta S, Ueno Y, Ishii M, Igarashi Y. Dynamic transition of a methanogenic population in response to the concentration of volatile fatty acids in a thermophilic anaerobic digester. Appl. Environ. Microb. 2006;72:1623-1630.

52. Merlino G, Rizzi A, Schievano A, et al. Microbial community structure and dynamics in two-stage vs single-stage thermophilic anaerobic digestion of mixed swine slurry and market bio-waste. Water Res. 2013;47:1983-1995.
53. Khelaifia S, Drancourt M. Susceptibility of archaea to antimicrobial agents: Applications to clinical microbiology. Clin. Microbiol. Infec. 2012;18:841-848.

54. Kandler O, Konig H. Cell wall polymers in Archaea (Archaebacteria). Cell. Mol. Life Sci. 1998;54:305-308.

55. Kohanski MA, Dwyer DJ, Collins JJ. How antibiotics kill bacteria: From targets to networks. Nat. Rev. Microbiol. 2010;8:423-435.

56. Tao W, Zhang X, Zhao F, et al. High levels of antibiotic resistance genes and their correlations with bacterial community and mobile genetic elements in pharmaceutical wastewater treatment bioreactors. Archaea 2016;11:1-17. 